\title{
A Fitting height lemma and its applications
}

\author{
M. YAsir KIZMAZ(i)
}

\begin{abstract}
Let $A$ be a group acting on a solvable group $G$ and let $N$ be an $A$-invariant normal subgroup of $G$ such that $[G, A] \nsubseteq N$. We prove the inequality $h([G, A]) \leq h([G, A] N / N)+h([N, A])$ where $h(G)$ denotes the Fitting height of $G$. As an application of this result, we obtain several Fitting height inequalities. A new concept "fixed point free separability" and a new characteristic subgroup $Y(G)$ is defined and used in order to prove some further results about the Fitting height of a group. In the last section, a new characterization of solvable groups is given: a group $G$ is solvable if and only if it is fixed point free separable.
\end{abstract}

Mathematics Subject Classification. 20D10, 20 D20.

Keywords. Fitting height, Solvable groups, Fixed point free separability.

1. Introduction. All groups considered in this paper are finite. Notation and terminology are standard as in [5]. Let $G$ be a solvable group and let $F(G)$ denote the Fitting subgroup of $G$. Set $F_{0}(G)=1, F_{1}(G)=F(G)$ and define inductively $F_{i+1}(G)$ as the full inverse image of $F\left(G / F_{i}(G)\right)$ in $G$. The smallest natural number $n$ such that $F_{n}(G)=G$ is called the Fitting height (nilpotent length) of $G$ and is denoted by $h(G)$. Note that the Fitting height of the trivial group is zero and the Fitting height of a nontrivial nilpotent group is 1 . In that sense, we can consider the Fitting height as a measure of how far away our group is from being nilpotent. It is well known that every normal nilpotent subgroup of $G$ is contained in $F(G)$, and more generally every normal nilpotent subgroup of $G$ with Fitting height $k$ is contained in $F_{k}(G)$.

Let $A$ be a group acting on a solvable group $G$ via automorphisms and $l(A)$ denote the number of not necessarily distinct primes whose product is $|A|$. The problem of finding a bound on the Fitting height of $G$ in terms of $l(A)$ and $l\left(C_{G}(A)\right)$ are studied by several authors. We refer to a comprehensive survey [4] by Khukhro for extensive discussions of results under this topic. 
If $N$ is a normal subgroup of a solvable group $G$, then one can deduce the well known inequality $h(G) \leq h(G / N)+h(N)$. If $N$ is also $A$-invariant, the following lemma shows that we can say more:

Lemma A. Let $A$ be a group acting on a solvable group $G$ via automorphisms. Assume that $N$ is an $A$-invariant normal subgroup of $G$ such that $[G, A] \nsubseteq N$. Then the following inequality is satisfied:

$$
h([G, A]) \leq h([G, A] N / N)+h([N, A]) .
$$

Corollary B. Let $A$ be a group acting on a solvable group $G$ via automorphisms and let $N$ be an $A$-invariant normal subgroup of $G$. If $[G, A]=G$, then we have

$$
h(G) \leq h(G / N)+h([N, A]) .
$$

Proof. The inequality clearly holds if $N=G$, so assume $N \neq G$. Then the result follows by Lemma A.

We observe that our lemma supplies extra information when $[N, A]<N$. Thus, we shall introduce a new characteristic subgroup $Y(G)$ of $G$ such that $[Y(G), A]<Y(G)$ under suitable conditions.

Definition 1.1. Let $A$ be a group acting via automorphisms on $G$. Set

$$
\mathcal{Y}_{A}(G)=\left\{X \leq G \mid X \text { is characteristic in } G \text { and } C_{G / X}(A)=1\right\} .
$$

We define $Y_{A}(G)$, the fixed point free residual of $G$ with respect to $A$, as the intersection of all members of $\mathcal{Y}_{A}(G)$.

We shall simply write $\mathcal{Y}(G)$ and $Y(G)$ when the group $A$ is obvious from the context. Note that $\mathcal{Y}(G)$ is nonempty as $G \in \mathcal{Y}(G)$. By the definition, it is clear that $Y(G)$ is a characteristic subgroup of $G$.

Let $\mathcal{Y}(G)=\left\{X_{i} \mid i=1, \ldots, n\right\}$ and let $\phi$ be a homomorphism from $G$ to $\prod_{i=1}^{n} G / X_{i}$ with $\phi(g)=\left(g X_{1}, g X_{2}, \ldots, g X_{n}\right)$. Then clearly we have $\operatorname{Ker}(\phi)=$ $Y(G)$. Note that $G / X_{i}$ is $A$-invariant for each $i$ as $X_{i}$ is characteristic in $G$. Then $A$ also acts via automorphisms on $\prod_{i=1}^{n} G / X_{i}$. The map $\phi$ satisfies the equality $\phi\left(g^{a}\right)=\phi(g)^{a}$ for all $g \in G$ and $a \in A$. Clearly, we have $C_{\phi(G)}(A)=1$ since $\phi(G) \leq \prod_{i=1}^{n} G / X_{i}$. This shows that $C_{G / Y(G)}(A)=1$ as $\phi\left(g^{a}\right)=\phi(g)^{a}$. Hence, we see that $Y(G) \in \mathcal{Y}(G)$, that is, $Y(G)$ is the smallest characteristic subgroup of $G$ such that the action of $A$ on $G / Y(G)$ is fixed point free.

Definition 1.2. Let $A$ be a group acting on a group $G$ via automorphisms. An $A$-invariant subnormal series $\mathcal{A}$ of $G$

$$
1=N_{k} \unlhd N_{1} \unlhd \cdots \unlhd N_{0}=G
$$

is called a fixed point free series of $G$ with respect to $A$ if the action of $A$ on each quotient $N_{i} / N_{i+1}$ is either trivial or fixed point free. Now let $\mathcal{A}$ be a fixed point free series of $G$. If the factors on which the action of $A$ is fixed point free are not consecutive in $\mathcal{A}$, then $\mathcal{A}$ is said to be a semistrong fixed point free series of $G$ (with respect to $A$ ). In addition to that, if factors on which the action of $A$ is trivial are not consecutive, then it is called a strong fixed point free series of $G$ (with respect to $A$ ). 
Definition 1.3. We say that $G$ is (semistrongly, strongly) fixed point free separable with respect to $A$ if there is a (semistrong, strong) fixed point free series $\mathcal{A}$ of $G$. The number of the nontrivial factors of $\mathcal{A}$ on which the action of $A$ is fixed point free is called the fixed point free length of $\mathcal{A}$, denoted by $\mathfrak{f}(\mathcal{A})$.

Definition 1.4. Let $\mathfrak{A}$ be the set of all fixed point free series of $G$ with respect to $A$. We define the fixed point free length of $G$ with respect to $A$ as

$$
\min \{\mathfrak{f}(\mathcal{A}) \mid \mathcal{A} \in \mathfrak{A}\}
$$

and it will be denoted by $\mathfrak{f}(G, A)$.

Definition 1.5. A group $G$ is said to be (semistrongly, strongly) fixed point free separable if it is (semistrongly, strongly) fixed point free separable with respect to each subgroup of $A u t(G)$.

Theorem C. Let $A$ be a group acting on a solvable group $G$ via automorphisms where $(|A|,|G|)=1$. Then $G$ is strongly fixed point free separable with $\mathfrak{f}(G, A) \leq l\left(C_{G}(A)\right)+1$.

Theorem D. Let $A$ be a group acting on a solvable group $G$ via automorphisms where $(|A|,|G|)=1$. Let $\mathcal{A}: 1=N_{k}<\cdots<N_{1}<N_{0}=G$ be a fixed point free series of $G$ and $T_{1}, T_{2}, \ldots, T_{m}$ be all the factors of $\mathcal{A}$ on which the action of $A$ is fixed point free. Then the following holds:

$$
h([G, A]) \leq \sum_{i=1}^{m} h\left(T_{i}\right) .
$$

The above theorem simply says that the Fitting height of $[G, A]$ is only bounded by the Fitting heights of the factors on which the action of $A$ is fixed point free, and we may ignore the ones on which $A$ acts trivially. Clearly, in the above sum $m=\mathfrak{f}(\mathcal{A})$ where $\mathcal{A}$ is the given fixed point free series of $G$. Together with Theorem $\mathrm{C}$, the previous theorem has a nice application: They supply a very short and representation theory free proof to the following well known result.

Theorem 1.6. Let $A$ be a group of prime order acting on a solvable group $G$ by automorphisms where $(|A|,|G|)=1$. Then the Fitting height of $G$ is at most $l\left(C_{G}(A)\right)+1$.

Proof. Write $N=[G, A]$ and $C=C_{N}(A)$. Clearly we have $\left|C_{G}(A)\right|=\mid G$ : $N|| C \mid$ as $G=N C_{G}(A)$, and so $l\left(C_{G}(A)\right)=l(G / N)+l(C)$. Now consider a fixed point free series $\mathcal{A}$ of $N$ such that $\mathfrak{f}(\mathcal{A})$ is as minimal as possible. Note that $[N, A]=N$. Then we have

$$
h(N) \leq \sum_{i=1}^{m} h\left(T_{i}\right)=m
$$

by Theorem $\mathrm{D}$. The equality holds as each $T_{i}$ must be nilpotent due to the Thomson theorem (see [5, Theorem 6.22]). On the other hand, $m=\mathfrak{f}(N, A) \leq$ $l(C)+1$ by applying Theorem $\mathrm{C}$ to $N$, and so $h(N) \leq l(C)+1$. It follows that 


$$
h(G) \leq h(G / N)+h(N) \leq l(G / N)+l(C)+1=l\left(C_{G}(A)\right)+1
$$

To be precise, we should note that [5, Theorem 6.22] has also a representation free proof.

setcountertheo4

Theorem E. Let $A$ be a group acting on a solvable group $G$ via automorphisms. Assume that $A$ is either cyclic or a p-group. Then $G$ is semistrongly fixed point free separable.

Let $A$ be a group acting on $G$ via automorphisms. We set $[G, A, \ldots, A]_{1}=$ $[G, A]$ and recursively define $[G, A, \ldots, A]_{n}=\left[[G, A, \ldots, A]_{n-1}, A\right]$ for $n \geq 2$. Clearly, there exists $m \in \mathbb{Z}$ such that $[G, A, \ldots, A]_{m}=[G, A, \ldots, A]_{m+1}$ as our groups are finite. We denote the repeated final commutator $[G, A, \ldots, A]_{m}$ by $[G, A, \ldots, A]_{\infty}$.

Theorem F. Let $A$ be a group acting on a solvable group $G$ via automorphisms. Write $K=[G, A, \ldots, A]_{\infty}$. Let $\mathcal{A}: 1=N_{k}<\cdots<N_{1}<N_{0}=G$ be a fixed point free series of $G$ and $T_{1}, T_{2}, \ldots, T_{m}$ be all the factors of $\mathcal{A}$ on which the action of $A$ is fixed point free. Then the following hold;

$$
h(K) \leq m+\sum_{i=1}^{m} h\left(T_{i}\right) \text { and } h([G, A]) \leq 1+m+\sum_{i=1}^{m} h\left(T_{i}\right) .
$$

The above theorem shows that an analogue of Theorem $\mathrm{D}$ is correct for the noncoprime case with extra term " $m+1$ ". Although Theorem E guarantees the existence of a semistrong fixed point series $\mathcal{A}$ of $G$ in the case that $A$ is cyclic or a $p$-group, unfortunately, it does not supply a bound for $\mathfrak{f}(\mathcal{A})$, and so it is unclear whether $\mathfrak{f}(G, A)$ is bounded in terms of $l\left(C_{G}(A)\right)$ and $l(A)$.

Question 1.7. Under the hypothesis of Theorem $E$, is $\mathfrak{f}(G, A)$ bounded by a function of $|A|$ and $\left|C_{G}(A)\right|$ ?

Let $A=\langle\varphi\rangle$ be a cyclic group acting on a solvable group $G$. In that case, the problem of finding a bound for $h(G)$ in terms of $|A|$ and $\left|C_{G}(A)\right|$ is still open (see the Kourovka Notebook [7, 13.8(a)]). However, there are some nice bounds proved in the case that the action is fixed point free, that is, when $C_{G}(A)=1$ : Under some further assumptions on the structure of $A$ and $G$, a linear bound has been obtained for $h(G)$ depending on $l(A)$ in $[2,3]$, and [8]. A current article established a quadratic bound $h(G) \leq 7 l(A)^{2}$ without any further assumptions on $A$ or $G$ (see [6, Corollary 1.2]). Since Theorem F creates a bridge between the general case and the fixed point free case, we might hope to attack the general case by using Theorem $\mathrm{F}$ and those results for the fixed point free case. Indeed, the following theorem shows that the open problem could be solved if Question 1.7 has an affirmative answer.

Theorem G. Let $A=\langle a\rangle$ be a cyclic group acting on a solvable group $G$ via automorphisms. If $\mathfrak{f}(G, A)$ is bounded by a function of $|A|$ and $\left|C_{G}(A)\right|$, then so is $h(G)$. 
In the following theorem, we obtain a new characterization of solvable groups.

Theorem H. A group $G$ is solvable if and only if it is fixed point free separable.

\section{The proofs of Theorems $\mathrm{C}$ and $\mathrm{D}$.}

Lemma 2.1. Let $G$ be a solvable group and $N$ be a proper central subgroup. Then $h(G / N)=h(G)$.

Proof of Lemma $A$. Let $k$ be the Fitting height of $[N, A]$. Then we have $[N, A] \leq$ $F_{k}(N)$ as $[N, A]$ is normal in $N$ and $F_{k}(N)$ is the largest normal subgroup of $N$ whose Fitting height is $k$. Clearly, $F_{k}(N) \unlhd G$.

Note that $[N, G, A] \leq[N, A] \leq F_{k}(N)$ and $[A, N, G]=[N, A, G] \leq$ $\left[F_{k}(N), G\right] \leq F_{k}(N)$. Then we have $[G, A, N] \leq F_{k}(N)$ by the three subgroups lemma.

Now write $\bar{G}=G / F_{k}(N)$. Then we have $[[\bar{G}, A], \bar{N}]=1$ by the argument above. Thus,

$$
\bar{N} \cap[\bar{G}, A] \leq Z([\bar{G}, A]) .
$$

Moreover, $\bar{N} \cap[\bar{G}, A]<[\bar{G}, A]$ as $[G, A] \nsubseteq \subseteq N$ by the hypothesis. Then we see that $h([\bar{G}, A] / \bar{N} \cap[\bar{G}, A])=h([\bar{G}, A])$ by Lemma 2.1 .

Consequently, we obtain

$$
h([G, A] N / N)=h([\bar{G}, A] \bar{N} / \bar{N})=h([\bar{G}, A] / \bar{N} \cap[\bar{G}, A])=h([\bar{G}, A]) .
$$

The first three equalities hold as they are isomorphic groups and the last equality follows from the previous paragraph. have

Clearly, $h([G, A]) \leq h\left([G, A] F_{k}(N)\right) \leq h([\bar{G}, A])+h\left(F_{k}(N)\right)$, and so we

$$
h([G, A]) \leq h([G, A] N / N)+h([N, A])
$$

as desired.

Lemma 2.2. Let $A$ be a group acting via automorphisms on $G$. Then $Y(Y(G))=$ $Y(G)$.

Proof. Assume the contrary, that is, $Y(Y(G))<Y(G)$. Notice that $Y(Y(G))$ is also characteristic in $G$. Set $\bar{G}=G / Y(Y(G))$. By the minimality of $Y(G)$, $C_{\bar{G}}(A) \neq 1$. Clearly, we also have $C_{\overline{Y(G)}}(A)=1$. That forces that $C_{\bar{G} / \overline{Y(G)}}(A) \neq$ 1. Set $\overline{\bar{K}}=C_{\bar{G} / \overline{Y(G)}}(A)$. Then $\overline{Y(G)}<\bar{K}$ with $[\bar{K}, A] \leq \overline{Y(G)}$. Thus, $[K, A] \leq$ $Y(G)$. Hence, $1<K / Y(G) \leq C_{G / Y(G)}(A)$, which is a contradiction.

Lemma 2.3. Let $A$ be a group acting on a nontrivial solvable group $G$ via automorphisms such that $[G, A]=G$. Assume that one of the following holds:

(a) $(|A|,|G|)=1$.

(b) $A$ is a p-group or a cyclic group.

Then the inequality $Y(G) \leq G^{\prime}<G$ holds. Moreover, $[Y(G), A]<Y(G)$ holds unless $Y(G)$ is the trivial subgroup. 
Proof. We have $G^{\prime}<G$ as $G$ is a nontrivial solvable group. Write $\bar{G}=G / G^{\prime}$.

First assume that $(|A|,|G|)=1$. Note that $\bar{G}=[\bar{G}, A] \times C_{\bar{G}}(A)$. Since $[\bar{G}, A]=[G, A] G^{\prime} / G^{\prime}=\bar{G}$, we see that $C_{\bar{G}}(A)=1$. Then we have $Y(G) \leq G^{\prime}$. Thus, $Y(G)$ is proper in $G$.

Let $A$ be a $p$-group. First we claim that $p$ does not divide the order of $\bar{G}$. Assume the contrary. Let $\bar{P}$ be a Sylow $p$-subgroup of $\bar{G}$, and write $\bar{G}=\bar{P} \times \bar{H}$ where $\bar{H}$ is the $p^{\prime}$-part. Clearly we have $[\bar{P}, A]<\bar{P}$ as $A$ is also a $p$-group. Then we get $[\bar{G}, A]=[\bar{P}, A] \times[\bar{H}, A] \leq[\bar{P}, A] \times \bar{H}<\bar{G}$, which is not the case. Thus, the action of $A$ on $\bar{G}$ is coprime and hence, $C_{\bar{G}}(A)=1$ as $[\bar{G}, A]=\bar{G}$. It follows that $Y(G) \leq G^{\prime}<G$.

Let $A=\langle a\rangle$ be a cyclic group. Let $\Phi: \bar{G} \rightarrow \bar{G}$ with $\Phi(\bar{g})=[\bar{g}, a]$. It is easy to see that $\Phi$ is a homomorphism with $\Phi(\bar{G})=[\bar{G}, a]=[\bar{G}, A]=\bar{G}$. Thus, $\operatorname{Ker}(\Phi)=C_{\bar{G}}(A)=1$. It follows that $Y(G) \leq G^{\prime}$.

Now assume that $Y(G)$ is nontrivial. If $[Y(G), A]=Y(G)$, then $Y(Y(G))<$ $Y(G)$ by the previous part, which is not possible by Lemma 2.2. Thus, $[Y(G), A]$ $<Y(G)$ unless $Y(G)$ is the trivial subgroup.

Proof of Theorem $C$. Set $N_{0}=G$. Define a series of $G$ recursively by

$$
N_{i+1}= \begin{cases}{\left[N_{i}, A\right]} & \text { if }\left[N_{i}, A\right] \neq N_{i}, \\ Y\left(N_{i}\right) & \text { if }\left[N_{i}, A\right]=N_{i}\end{cases}
$$

Note that $N_{i+1}$ is proper in $N_{i}$ unless $N_{i}$ is the trival group by Lemma 2.3, which forces that $N_{k}=1$ for some $k$. It is also clear that each $N_{i}$ is $A$-invariant and $N_{i+1}$ is normal in $N_{i}$. Moreover, $A$ acts on each factor $N_{i} / N_{i+1}$ trivially or fixed point freely by our settings, and so it is a fixed point free series of $G$.

If $A$ acts on the factor $N_{i} / N_{i+1}$ trivially, then $A$ acts on the next factor $N_{i+1} / N_{i+2}$ fixed point freely and vice versa due to the identity $[G, A, A]=$ $[G, A]$ in a coprime action and the identity $Y(Y(G))=Y(G)$ (see Lemma 2.2). Let us assume that there are $n$ factors on which the action of $A$ is trivial. It follows that there are at most $n+1$ factors on which the action of $A$ is fixed point free, and hence $\mathfrak{f}(G, A) \leq n+1$.

To finish the proof, we need to show that $n \leq l\left(C_{G}(A)\right)$. Let $S_{1}, S_{2}, \ldots, S_{n}$ be all the factors on which the action of $A$ is trivial. Note that the equality

$$
\left|C_{G}(A)\right|=\prod_{i=1}^{n}\left|S_{i}\right|
$$

is immediate from the identity $\overline{C_{G}(A)}=C_{\bar{G}}(A)$ in a coprime action. Thus, we obtain that $n \leq l\left(C_{G}(A)\right)$, which completes the proof.

Proof of Theorem $D$. We proceed by induction on the order of $G$. First assume that $A$ acts trivially on $N_{0} / N_{1}=G / N_{1}$. Then $\mathcal{B}: 1=N_{k}<\cdots<N_{2}<N_{1}$ is a fixed point free series of $N_{1}$ such that $T_{1}, T_{2}, \ldots, T_{n}$ appear in $\mathcal{B}$. By induction applied to $N_{1}$, we have $h\left(\left[N_{1}, A\right]\right) \leq h\left(T_{1}\right)+h\left(T_{2}\right)+\cdots+h\left(T_{n}\right)$. Note that $[G, A] \leq N_{1}$ as $A$ acts trivially on $G / N_{1}$, and so we obtain $[G, A, A] \leq\left[N_{1}, A\right] \leq$ $[G, A]$. It follows that $\left[N_{1}, A\right]=[G, A]$ as $[G, A, A]=[G, A]$ in a coprime action, which completes the proof for this case. 
Now assume that $A$ acts fixed point freely on $G / N_{1}$, and without loss of generality set $T_{1}=G / N_{1}$. Then we clearly have $[G, A] \nsubseteq N_{1}$. It follows that

$$
h([G, A]) \leq h\left([G, A] N_{1} / N_{1}\right)+h\left(\left[N_{1}, A\right]\right)
$$

by Lemma A. Note that $[G, A] N_{1} / N_{1} \leq T_{1}$, and so

$$
h([G, A]) \leq h\left(T_{1}\right)+h\left(\left[N_{1}, A\right]\right) .
$$

Moreover, $\mathcal{B}: 1=N_{k}<\cdots<N_{2}<N_{1}$ is a fixed point free series of $N_{1}$ such that $T_{2}, \ldots, T_{n}$ appear in $\mathcal{B}$. Thus, we get $h\left(\left[N_{1}, A\right]\right) \leq h\left(T_{2}\right)+\cdots+h\left(T_{n}\right)$ by induction applied to $N_{1}$. Then the result follows.

\section{The proofs of Theorems $\mathbf{E}$ and $\mathbf{F}$.}

Proof of Theorem E. Set $N_{0}=G$. Define a series $\mathcal{A}$ of $G$ recursively by

$$
N_{i+1}= \begin{cases}{\left[N_{i}, A\right]} & \text { if }\left[N_{i}, A\right] \neq N_{i}, \\ Y\left(N_{i}\right) & \text { if }\left[N_{i}, A\right]=N_{i} .\end{cases}
$$

Then the proof follows as in the proof of Theorem $\mathrm{C}$ by applying Lemma 2.3(b). On the other hand, if two factors on which the action of $A$ is fixed point free are consecutive, then we must have $Y\left(Y\left(N_{i}\right)\right)<Y\left(N_{i}\right)$ for some $i$, which is impossible by Lemma 2.2. Thus, $\mathcal{A}$ is a semistrong fixed point free series of $G$.

Lemma 3.1. Let $A$ be a group acting on a solvable group $G$ via automorphisms. Set $K=[G, A, \ldots, A]_{\infty}$. Then $h([G, A]) \leq h(K)+1$.

Proof. Let $h(K)=k$. Then $K \leq F_{k}(G)$ as $K$ is subnormal in $G$. Then we have $\left[G / F_{k}(G), A, \ldots, A\right]_{\infty}=1$, which implies that $\left[G / F_{k}, A\right]$ is nilpotent by the Hall theorem [5, Theorem 4.27]. Thus we get that $[G, A] F_{k} / F_{k}$ is nilpotent which yields that $h([G, A]) \leq k+1$.

Corollary 3.2. Let $A$ be a group acting on a solvable group $G$ via automorphisms. Then the following hold:

(a) If $N$ is an A-invariant normal subgroup of $G$ such that $[G, A] \nsubseteq N$, then

$$
h([G, A]) \leq h([G, A] N / N)+h\left([N, A, \ldots, A]_{\infty}\right)+1 .
$$

(b) If $[G, A]=G$, then

$$
h(G) \leq h(G / N)+h\left([N, A, \ldots, A]_{\infty}\right)+1
$$

holds for an arbitrary $A$-invariant normal subgroup $N$ of $G$.

Proof. It directly follows from Lemma A, Corollary B, and Lemma 3.1.

Lemma 3.3. Let $A$ be a group acting via automorphisms on a group $G$. Let $K$, $U$, and $V$ be $A$-invariant subgroups of $G$ such that $V \unlhd U$. If $A$ acts trivially or fixed point freely on $U / V$, then $A$ acts trivially or fixed point freely on $U \cap K / V \cap K$, respectively. Moreover, $U \cap K / V \cap K$ is isomorphic to a subgroup of $U / V$.

We skip the elementary proof of the above lemma. 
Proof of Theorem $F$. We shall prove that $h(K) \leq m+\sum_{i=1}^{m} h\left(T_{i}\right)$ where $K=$ $[G, A, \ldots, A]_{\infty}$ by induction on the order of $G$. Assume first that $[G, A]=G$, that is, $K=G$. Clearly, $A$ acts fixed point freely on $N_{0} / N_{1}$, and so we may set $T_{1}=N_{0} / N_{1}$. We have $h(G) \leq h\left(T_{1}\right)+h\left(\left[N_{1}, A, \ldots, A\right]_{\infty}\right)+1$ by Corollary $3.2(\mathrm{~b})$.

Then $\mathcal{B}: 1=N_{k}<\cdots<N_{2}<N_{1}$ is a fixed point free series of $N_{1}$ such that $T_{2}, \ldots, T_{n}$ appear in $\mathcal{B}$. By induction applied to $N_{1}$, we get

$$
h\left(\left[N_{1}, A, \ldots, A\right]_{\infty}\right) \leq m-1+\sum_{i=2}^{m} h\left(T_{i}\right) .
$$

Thus, $h(K)=h(G) \leq m+\sum_{i=1}^{m} h\left(T_{i}\right)$.

Now assume $[G, A]<G$. Set $M_{i}=K \cap N_{i}$ for each $i$. Then $\mathcal{C}: 1=$ $M_{k} \unlhd M_{1} \unlhd \cdots \unlhd M_{0}=K$ is a fixed point free series of $K$ such that $T_{1}^{*}, T_{2}^{*}, \ldots, T_{m}^{*}$ are the all factors of $\mathcal{C}$ on which the action of $A$ is fixed point free where $T_{i}^{*}$ is isomorphic to a subgroup of $T_{i}$ by Lemma 3.3. Thus, we get $h(K) \leq$ $m+\sum_{i=1}^{m} h\left(T_{i}^{*}\right) \leq m+\sum_{i=1}^{m} h\left(T_{i}\right)$ by the inductive argument, which proves the first inequality.

Consequently, we get $h([G, A]) \leq h(K)+1 \leq 1+m+\sum_{i=1}^{m} h\left(T_{i}\right)$ by Lemma 3.1 , which proves the second inequality.

\section{The proofs of Theorems $G$ and $H$.}

Proof of Theorem $G$. We see that $G$ is semistrongly fixed point free separable with respect to $A$ by Theorem E. Let $\mathcal{A}$ be a fixed point free series of $G$ such that $\mathfrak{f}(\mathcal{A})=\mathfrak{f}(G, A)$. By Theorem $\mathrm{F}$, we have $h([G, A]) \leq 1+m+\sum_{i=1}^{m} h\left(T_{i}\right)$ where $m=\mathfrak{f}(G, A)$. We know that $h\left(T_{i}\right) \leq 7 l(A)^{2}$ for $i=1, \ldots, m$ by $[6$, Corollary 1.2], and so

$$
h([G, A]) \leq 1+m+m\left(7 l(A)^{2}\right) .
$$

Since $m=\mathfrak{f}(G, A)$ is bounded by a function of $|A|$ and $\left|C_{G}(A)\right|$ by the hypothesis, $h([G, A])$ is also bounded in terms of $|A|$ and $\left|C_{G}(A)\right|$. Note that $h(G) \leq h([G, A])+h(G /[G, A])$. To finish the proof, we shall show that $h(G /[G, A]) \leq\left|C_{G}(A)\right|$. Let $\Phi: G \rightarrow G$ with $\Phi(g)=[g, a]$ for all $g \in G$. Note that $\Phi$ is a crossed homomorphism, and so $|G| /|\operatorname{Ker}(\Phi)|=|G| /\left|C_{G}(A)\right|=$ $|\operatorname{Im}(\Phi)| \leq|[G, A]|$ which yields $|G:[G, A]| \leq\left|C_{G}(A)\right|$. In particular, we have $h(G /[G, A]) \leq\left|C_{G}(A)\right|$, which proves the claim.

Lemma 4.1. Let $A$ be a group acting on $G$ via automorphisms and $N$ be an $A$-invariant normal subgroup of $G$. Then $G$ is fixed point free separable with respect to $A$ if both $G / N$ and $N$ are fixed point free separable with respect to A.

The proof of the above lemma is routine and elementary, so we skip the proof. We also need the next theorem in the proof of Theorem $\mathrm{H}$.

Theorem 4.2. Let $A$ be a nilpotent group acting on a group $G$ via automorphisms. If $G$ is fixed point free separable with respect to $A$, then $[G, A]$ is solvable. 
Proof. We proceed by induction on the order of $G$. Let $N=[G, A]$. Note that $N$ is also fixed point free separable with respect to $A$ by Lemma 3.3.

First assume that $[N, A]<N$. Then we also have $N<G$, and so $[N, A]$ is solvable by induction applied to $N$. Let $S$ be the largest normal subgroup of $N$. Since $[N, A]$ is normal in $N$, we get that $[N, A]=[G, A, A] \leq S$. Moreover, $S \unlhd G$ as $N \unlhd G$ and $S$ is characteristic in $N$. Set $\bar{G}=G / S$. Then we have that $[\bar{G}, A, A]=1$, which yields that $[\bar{G}, A]=N / S$ is abelian by $[5$, Corollary 4.23]. It follows that $N=[G, A]$ is solvable.

Now assume that $N=[N, A]$. Let $\mathcal{B}: 1=N_{k}<\cdots<N_{2}<N_{1}=N$ be a fixed point free series of $N$. Clearly, we may assume that $N$ is not trivial, so $k \geq 2$ and the action of $A$ on $N / N_{2}$ is fixed point free. We see that $N / N_{2}$ is solvable by $[1$, Poposition 1.2] as $A$ is nilpotent. Thus, we need to show that $N_{2}$ is solvable to finish the proof.

By induction applied to $N_{2}$, we get that $\left[N_{2}, A\right]$ is solvable. Let $S$ be the largest normal solvable subgroup of $N_{2}$. Then $\left[N_{2}, A\right] \leq S$ and $S$ is normal in $N$. Now set $\bar{N}=N / S$. Note that $\left[\bar{N}_{2}, A\right]=1$. Thus, $\left[\bar{N}, \bar{N}_{2}, A\right]=\left[\bar{N}_{2}, A, \bar{N}\right]=$ 1 which yields that $\left[A, \bar{N}, \bar{N}_{2}\right]=1$ by the three subgroups lemma. Since $[N, A]=N$, we get $\left[\bar{N}, \bar{N}_{2}\right]=1$, that is, $\left[N, N_{2}\right] \leq S$. Thus, $N_{2}$ is solvable, which completes the proof.

Proof of Theorem $H$. We first show that if $G$ is solvable, then $G$ is fixed point free separable. Let $G$ be a minimal counter example to the claim. Then there exists $A \leq \operatorname{Aut}(G)$ such that $G$ is not fixed point free separable with respect to $A$.

Assume that there exists an $A$-invariant nontrivial proper normal subgroup $N$ of $G$. Then both $G / N$ and $N$ are fixed point free separable by the minimality of $G$. In particular, they are fixed point free separable with respect to $A$. This implies that $G$ is fixed point free separable with respect to $A$ by Lemma 4.1. This contradiction shows that $G$ has no such subgroup.

Note that we have $G^{\prime}<G$ as $G$ is solvable, and so we see that $G^{\prime}=1$ by the previous paragraph, that is, $G$ is abelian. Then $C_{G}(A)$ is a normal $A$-invariant subgroup of $G$, which forces that $C_{G}(A)=G$ or $C_{G}(A)=1$ by the previous paragraph. This contradiction completes the proof.

Now assume that $G$ is fixed point free separable. Let $S$ be the largest normal solvable subgroup of $G$. Let $g \in G$ and set $A=\langle g\rangle$. By hypothesis, $G$ is fixed point free separable with respect to $A$. Clearly $A$ is nilpotent. It follows that $[G, A]=[G, g]$ is solvable by Theorem 4.2 . Then we have $[G, g] \leq S$ as $[G, g] \unlhd G$. It follows that $G^{\prime}$ is solvable, and so $G$ is solvable.

5. Examples and final remarks. The following example shows that the assumption $[G, A] \nsubseteq N$ in Lemma A is necessary.

Example 5.1. Let $G=S_{4}$, the symmetric group on four letters, and $A=$ $\langle(1,2,3)\rangle$. Now set $N=A_{4}$. Then we see that $[G, A]=A_{4}=N$ and $[N, A]=$ $V_{4}$, which is the Klein four group. However,

$$
2=h([G, A]) \not \leq h([G, A] N / N)+h([N, A])=h(1)+h\left(V_{4}\right)=1 .
$$

Now we shall observe that both of the inequalities in Theorem $\mathrm{F}$ are tight. 
Example 5.2. Let $G=S_{4}$ and $A=\langle(1,2)\rangle$. Then $\mathcal{A}: 1<\left[V_{4}, A\right]<V_{4}<$ $A_{4}<S_{4}$ is a fixed point free series of $G$ with respect to $A$ where $A_{4} / V_{4}$ is the only factor on which $A$ acts fixed point freely. Thus, $m=\mathfrak{f}(\mathcal{A})=1$. Moreover, $K=[G, A, \ldots A]_{\infty}=[G, A]=A_{4}$. Then

$$
2=h(K) \leq m+h\left(A_{4} / V_{4}\right)=2 .
$$

Now set $A=\langle(1,2)(3,4)\rangle$. Then $\mathcal{A}: 1<V_{4}<S_{4}$ is a fixed point free series of $G$ with respect to $A$ where $A$ acts trivially on all factors of $\mathcal{A}$. Thus, $m=\mathfrak{f}(\mathcal{A})=0$. Moreover, $[G, A]=V_{4}$ and

$$
1=h([G, A]) \leq 1+m=1 .
$$

Final remarks. The concept of fixed point free separability and its variations are linked to the theory of solvable groups powerfully as seen in the current article, and so it has potential for further development.

One can pose several natural questions that are arising from the current article: Let $A$ be a group acting on a solvable group $G$ via automorphisms. Is there any conceivable condition guaranteeing strong fixed point free separability of $G$ with respect to $A$ beside the coprimeness? Is there any bound for $\mathfrak{f}(G, A)$ in terms of $h\left(C_{G}(A)\right)$ and $l(A)$ if $(|A|,|G|)=1$ ? Moreover, Question 1.7 can be asked in a general way: Assume that $G$ is semistrongly fixed point free separable with respect to $A$. Is $\mathfrak{f}(G, A)$ bounded by a function of $|A|$ and $\left|C_{G}(A)\right| ?$

Lastly, we note that a subgroup $Y^{*}(G)$ similar to $Y(G)$ might be also useful for studying fixed point free series of $G$ although $Y(G)$ seems to be more practical to work with in the current article. Set

$\mathcal{Y}^{*}(G)=\left\{X \leq G \mid X\right.$ is $A$-invariant and normal in $G$ and $\left.C_{G / X}(A)=1\right\}$. We define $Y^{*}(G)$ as the intersection of all members of $\mathcal{Y}^{*}(G)$. Then it is routine to see that $Y^{*}(G)$ is the smallest $A$-invariant normal subgroup of $G$ such that $C_{G / Y^{*}(G)}(A)=1$.

Publisher's Note Springer Nature remains neutral with regard to jurisdictional claims in published maps and institutional affiliations.

\section{References}

[1] Belyaev, V.V., Hartley, B.: Centralizers of finite nilpotent subgroups in locally finite groups. Algebra i Logika 35, 389-410 (1996) (Russian). English transl. Algebra and Logic 35, 217-228 (1996)

[2] Ercan, G.: On a Fitting length conjecture without the coprimeness condition. Monatsh. Math. 167(2), 175-187 (2012)

[3] Ercan, G.: Güloğlu, İ.Ş.: Fixed point free action on groups of odd order. J. Algebra 320, 426-436 (2008)

[4] Evgeny, I.: Problems of bounding the p-length and Fitting height of finite soluble groups. J. Sib. Fed. Univ. Math. Phys. 6(4), 462-478 (2013)

[5] Isaacs, I.: Martin: Finite Group Theory. Graduate Studies in Mathematics, vol. 92. American Mathematical Society, Providence (2008) 
[6] Jabara, E.: The Fitting length of finite soluble groups II: fixed-point-free automorphisms. J. Algebra 487, 161-172 (2017)

[7] Khukhro, E.I., Mazurov, V.D.: Unsolved Problems in Group Theory. The Kourovka Notebook. arXiv:1401.0300v20 (2020)

[8] Turull, A., Character theory and length problems. In: Finite and Locally Finite Groups (Istanbul, 1994), 377-400, NATO Adv. Sci. Inst. Ser. C Math. Phys. Sci., 471. Kluwer Acad. Publ., Dordrecht (1995)

M. YAsir KizmaZ

Department of Mathematics

Bilkent University

06800 Bilkent Ankara

Turkey

e-mail: yasirkizmaz@bilkent.edu.tr

Received: 8 January 2021

Revised: 23 January 2021

Accepted: 8 February 2021. 